\title{
Structural Connectivity and Cortical Thickness Alterations in Transient Global Amnesia
}

\author{
(D). Hodel, (D)X. Leclerc, (D) M. Zuber, (D). Gerber, (D). Besson, (D) V. Marcaud, (DV. Roubeau, (D). Brasme, (DI. Ganzoui,
} (D)D. Ducreux, DJ.-P. Pruvo, (D) M. Bertoux, (D). Zins, and (D) R. Lopes

\begin{abstract}
BACKGROUND AND PURPOSE: Transient global amnesia (TGA) is a sudden onset of anterograde and retrograde amnesia. We aimed to assess differences in terms of cortical thickness and structural brain connectome between patients with TGA (at acute and delayed postrecovery stages) and matched controls.
\end{abstract}

MATERIALS AND METHODS: We report on 18 consecutive patients with TGA who underwent 3T MR imaging, including DTI and MPRAGE sequences, at the acute (mean delay postonset: 44 hours) and delayed post-recovery (mean delay: 35 days) stages. Structural connectome was assessed in patients with TGA and in 18 age- and sex-matched controls by using probabilistic fibertracking and segmentation of 164 cortical/subcortical structures ("nodes"). Connectivity graphs were computed and global network metrics were calculated. Network-based statistical analysis (NBS) was applied to compare patients with TGA at each stage with controls. We also compared cortical thickness between patients with TGA and healthy controls.

RESULTS: Global network metrics were not altered in patients with TGA. NBS-analysis showed structural connectome alterations in patients with TGA compared with controls, in core regions involving the limbic network, with 113 nodes and 114 connections (33 left intrahemispheric, 31 right intrahemispheric, and 50 interhemispheric connections) showing significantly decreased structural connectivity $(P<.05$ NBS corrected, $t$-values ranging from 3.03 to 8.73). Lower cortical thickness compared with controls was associated with these structural alterations in patients with TGA, involving the orbitofrontal, cingulate, and inferior temporal cortices. All the abnormalities were visible at both acute and delayed postrecovery stages.

CONCLUSIONS: Our preliminary study suggests there are structural abnormalities of the limbic network in patients with TGA compared with controls, including decreased structural connectivity and cortical thickness.

ABBREVIATIONS: TGA = transient global amnesia; TOF-MRA = time-of-flight MRA; VBM = voxel-based morphometry; AUC = area under the curve; $\mathrm{FDR}=$ false discovery rate; $\mathrm{NBS}=$ network-based statistical analysis

$\mathbf{T}$ ransient global amnesia (TGA) is characterized by a sudden onset of an anterograde and retrograde amnesia that lasts up to 24 hours. ${ }^{1}$ The pathogenesis of TGA remains unclear, however long-term cognitive performance is similar between patients with TGA and healthy controls. ${ }^{2}$ Hippocampal punctate hyperintensities can be observed in up to $70 \%$ of patients with TGA by using diffusion-weighted imaging. ${ }^{3}$ Such lesions are better detected on an optimized DWI sequence ${ }^{4,5}$ and may be related to a delayed neuronal loss within the hippocampus cornu ammonis 1-subfield (CA1-subfield). ${ }^{6}$

Received October 17, 2019; accepted after revision March 12, 2020.

From the Departments of Radiology (J.H., S.G., I.G., M.Z.), and Neurology (M.Z.,

V.M., V.R., H.B.), Saint Joseph Hospital, Paris, France; Department of Neuroradiology

(X.L., J.-P.P., R.L.), Roger Salengro Hospital, Lille, France; INSERM UMR S919 (M.Z.),

Université Paris Descartes, Sorbonne Paris Cité, Paris, France; Department of

Radiology (P.B.), Northwestern University, Feinberg School of Medicine, Chicago,

Illinois; Department of Neuroradiology (D.D.), Bicêtre Hospital, Kremlin-Bicêtre,

France; and University of Lille (M.B., R.L.), Inserm U1171, CHU Lille, F-59000, Lille,

France.
Several neuroimaging studies revealed more widespread abnormalities in patients with TGA. Using cortical morphometry, pre-existing structural abnormalities were reported in patients with TGA mainly involving the default mode network. $^{7,8}$ The thalamus and the prefrontal cortex have been reported to be functionally altered during TGA by using positron emission tomography assessment. ${ }^{9-11}$ Using single-photon emission CT, Jang et $\mathrm{al}^{12}$ found that anterior lesions on DWI were associated with hypoperfusion of the frontal and anterior temporal areas whereas posterior lesions were associated with

Please address correspondence to Jérôme Hodel, MD, PhD, Department of Radiology, Saint Joseph Hospital, Paris, France; e-mail: jerome.hodel@gmail.com; @ci2clab

Indicates article with supplemental on-line appendix and table.

Indicates article with supplemental on-line photos.

http://dx.doi.org/10.3174/ajnr.A6530 
hypoperfusion of the parietal, posterior temporal, occipital, and cerebellar areas. More recently, 2 resting-state functional MR imaging studies revealed a decreased functional connectivity in TGA. Peer et $\mathrm{al}^{13}$ showed functional alterations involving the episodic memory network including the frontal regions, cingulate cortex, and basal ganglia. Zidda et $\mathrm{al}^{14}$ demonstrated alterations of the executive and salience networks including frontal and prefrontal regions, cingulate gyrus, parietal, insular and hippocampus.

However, the connectivity alterations at the acute stage of TGA, and their evolution at delayed postrecovery stage, have not been investigated yet by brain morphometry or wholebrain structural connectome. Considering the clinical phenomenology of TGA and previous neuroimaging studies, we hypothesized that the structural connectome could also be altered in patients with TGA, particularly within the limbic network that includes the hippocampus, temporal pole, parahippocampal gyrus, amygdala, anterior and posterior cingulate gyrus, and medial and lateral orbitofrontal cortex. To test this hypothesis, we aimed to assess differences in terms of cortical thickness and structural brain connectome between patients with TGA (at acute and delayed postrecovery stages) and matched controls.

\section{MATERIALS AND METHODS Participants}

The Institutional Review Board approved this prospective study. Informed written consent was obtained according to ethical standard procedures. During a study inclusion of 2 years, the inclusion criteria were the following:

1) Right-handed patient.

2) Diagnosis of TGA according to Hodges and Warlow criteria ${ }^{1}$ including: a) presence of an anterograde amnesia that is witnessed by an observer, b) no clouding of consciousness or loss of personal identity, c) cognitive impairment limited to amnesia, d) no focal neurologic or epileptic signs, e) no recent history of head trauma or seizures, and f) resolution of symptoms within 24 hours.

3) No personal history of prior episodes of TGA, drug abuse, or neurologic or psychiatric disorders.

4) Neurologic examination considered as normal at acute and delayed postrecovery stages.

5) 3 T MRI available at both acute (less than 48 hours after onset) and delayed (between 30 to 40 days after onset) stages.

Exclusion criteria included: age under 18 years, contraindication for 3T MR imaging, brain lesion or motion artifacts detected on MR images. We excluded patients and controls in case of 1) white matter hyperintensities with a score of 2 or 3 according to the Fazekas scale, 2) acute or old brain infarct(s), 3) more than 3 microbleeds, or 4) brain tumor, because these brain lesions may have potentially altered the tractography of the fibers. Hippocampal hyperintensity was not exclusionary for patients with TGA.

The recruitment of healthy controls was based on clinical examination (including the Mini-Mental State Examination), socio-demographic factors, and MR imaging data. The healthy volunteers were selected from a cohort of healthy subjects once the cohort of patients with TGA was fully established. Indeed, each control was selected to correspond to a patient with TGA in terms of sex and age. The exclusion criteria applied to patients with TGA and controls. Both patients with TGA and healthy volunteers had no personal history of prior episodes of TGA, drug abuse, neurologic or psychiatric disorders, and had normal structural MR imaging.

\section{MR Imaging Acquisition}

Patients with TGA and healthy controls were scanned at the same site by using 3T MR imaging (Discovery MR750; GE Healthcare) with the same MR sequences: 1) 3D T1-weighted MPRAGE: TR/TE, 2530/1.74 ms; flip angle, $70^{\circ}$; matrix, 256 x 256; isotropic 1-mm voxels; acquisition time, 7 minutes; 2) singleshot echo-planar diffusion tensor imaging: TR/TE, 8080/83 ms; flip angle, $90^{\circ}$; matrix, $128 \times 128$; isotropic $2-\mathrm{mm}$ voxels; $10 \mathrm{~b}=$ $0 \mathrm{~s} / \mathrm{mm}^{2}$ images ("b0 images") and 64 directional gradients at $b=1000$ seconds $/ \mathrm{mm}^{2}$; acquisition time, 10 minutes. DTI trace images and apparent diffusion coefficient maps were systematically generated. In all patients and healthy controls, the MR imaging protocol also included axial T2-weighted, 3D FLAIR, time-of-flight MRA (TOF-MRA), and susceptibility-weighted MR images.

\section{Analysis of Conventional MR Images}

In each subject, 2 senior neuroradiologists (J.H. and X.L.) analyzed in consensus all the MR images available to ensure the lack of brain lesions, and in search of potential hippocampal lesions on DTI trace images and ADC maps.

\section{Image Pre-processing and Whole-Brain Tractography}

For each participant, MPRAGE images were segmented in 74 cortical regions and 8 subcortical regions per hemisphere by using FreeSurfer software (v5.3.0, http://surfer.nmr.mgh. harvard.edu/). Moreover, the cortical thickness map in native space was generated by FreeSurfer software. DTI data were preprocessed for eddy current distortions and motion artifacts by using FSL software (FMRIB Software Library; http://www. fmrib.ox.ac.uk/fsl/), and the MPRAGE image was nonlinearly registered to the mean b0 image by using ANTS software (http://stnava.github.io/ANTs/). Whole-brain fiber-tracking was performed by using the MRtrix3 software package (http:// www.mrtrix.org/). The algorithm generated 1,000,000 fibers of minimum length $20 \mathrm{~mm}$. All steps are detailed in On-line Appendix and were manually checked for errors by visual inspection. In this study, image preprocessing and analysis were performed without knowledge of the presence of hippocampal hyperintensities on DTI trace images.

\section{Network Construction}

The nodes of graph G represented the 164 brain ROIs and edges, the brain subcortical and cortical structure connections. For any pair of nodes I and J, an edge was added to $G$ if at least 1 fiber tract connected these 2 ROIs. Moreover, the edge was weighted by the sum of fiber tracts connecting these 2 ROIs divided by the volume of the 2 ROIs. Thus, for each subject, a 164 x 164 symmetric weighted graph was constructed.

AJNR Am J Neuroradiol 41:798-803 May 2020 www.ajnr.org 


\section{Statistical Analyses}

Demographic Data. Between-group difference in age and sex between controls and patients was assessed by student and $\chi^{2}$ tests, respectively.

Network Topology Analysis. The weighted graph G was thresholded at different levels of sparsities that ranged from $\mathrm{T}=10 \%$ to $35 \%$ by using increments of $1 \%$, in keeping the highest weighted edges. Then, further analysis was based on the binarized graph $\mathrm{G}_{\mathrm{T}}$ for each subject.

At each threshold, global network metrics were calculated by using GRETNA toolbox (https://www.nitrc.org/projects/ gretna/): clustering coefficient $\left(\mathrm{C}_{\mathrm{p}}\right)$, shortest path length $\left(\mathrm{L}_{\mathrm{p}}\right)$, global efficiency $\left(\mathrm{E}_{\mathrm{g}}\right)$, and local efficiency $\left(\mathrm{E}_{\mathrm{loc}}\right)$. The metrics were described by Rubinov et $\mathrm{al}^{15}$ The area under the curve (AUC) for each network metric was calculated to provide a summarized scalar for topologic organization of brain networks independent of a single threshold selection. ${ }^{16}$ Nonparametric permutation tests (20,000 permutations) were used to test between-group differences in AUC values of network metrics (ie, clustering coefficient, mean path length, local efficiency, and global efficiency). Patients with TGA between acute and delayed stages $(n=18)$ and with agematched controls $(n=18)$ were compared by using paired and 2 -sample $t$-tests, respectively. The results are presented at a statistical threshold of $P<.05$ (false discovery rate (FDR)-corrected).

Structural Connectivity Analysis. To further identify altered structural connections in patients with TGA between acute and delayed stages compared with controls, network-based statistical analysis (NBS) was used on the unthresholded corrected graph, which is a validated nonparametric statistical approach for controlling family-wise error in connectome analyses. ${ }^{17}$ NBS was applied to compare patients and controls by paired and 2-sample $t$-tests as follows: ${ }^{1}$ mean connectivity strength changes were calculated with permutation tests, ${ }^{2}$ network components of interconnected edges that survived a $p$-value of .005 uncorrected were retained, ${ }^{3}$ and the size of the largest cluster was calculated. To generate an empirical null distribution for evaluating the statistical significance of the cluster sizes, groups were randomly shuffled (20,000 permutations), then the largest cluster size null distribution was obtained by repeating steps 1,2 , and 3 . The significance level of altered components in the NBS analysis was set to .05 (NBS-corrected).

\section{Cortical Thickness and Subcortical Volumetric Analyses}

Because our purpose was to assess the between-group differences in cortical thickness and subcortical volumetry within brain regions associated with structural connectivity alterations, an ROI analysis was also performed. The mean cortical thickness of cortical ROI and volumetry of subcortical ROI were measured by FreeSurfer software and compared between patients with TGA at acute and delayed stages and controls to assess if connectivity alterations were associated with cortical thickness or subcortical volumetry differences between groups. Nonparametric permutation tests (20,000 permutations) were used to test between-group differences. The results are presented at a statistical threshold of $P<.05$ (FDR-corrected).

\section{RESULTS \\ Participants}

During the 2-year inclusion period, 24 consecutive patients were diagnosed with TGA in our institution. Of these 24 patients, 4 presented motion artifacts on MR imaging and MR imaging was not available at the delayed postrecovery stage in 2 . Finally, 18 patients with TGA (14 womens, mean age 64.5 years, range: $40-$ 80 years, all right-handed) were included in the study. All patients $(n=18)$ underwent 3T MR imaging (Discovery MR750) at both acute (mean scan time from onset: 44 hours, range: $24-51$ hours) and delayed postrecovery (mean scan time from onset: 35 days, range: 31-39 days) TGA stages. Characteristics of patients with TGA are shown in the Table.

In addition, 18 age and sex-matched, right-handed, healthy volunteers served as controls. The controls perfectly matched with patients in terms of sex (18 subjects including 14 [78\%] females) and were close in terms of age (the mean age of patients was $64 \pm 11$ years; it was $61 \pm 13$ years in controls). The comparison between patients with TGA and controls did not show any significant statistical difference for age $(P=.46)$ or $\operatorname{sex}(P=.1)$.

\section{Hippocampal Hyperintensities on DTI Trace Images}

Punctate hyperintensities were visible on DTI trace images in 12 of the 18 patients with TGA. Results are detailed in the Table. There were no abnormalities detected in controls. Postprocessing data showed no correlation between hippocampal hyperintensities and structural connectivity $(P=.38$, Mann-Whitney test) or volume of hippocampus ( $P=.61$, Mann-Whitney test).

\section{Network Topology}

No differences of the global network metrics were observed between patients with TGA at acute and delayed stages and healthy controls (Fig 1).

\section{Structural Connectivity}

NBS Comparing patients with TGA at Acute Phase and Healthy Controls. In comparison with controls, a cluster of 113 nodes and 114 connections (33 left intrahemispheric, 31 right intrahemispheric, and 50 interhemispheric connections) had significantly decreased structural connectivity (ie, decreased number of fiber tracts) $(P<.05$ NBS corrected, $\mathrm{t}$ values ranging from 3.03 to 8.73 ) in patients with TGA at acute phase (Fig $2 A$ and On-line Table).

In this widespread network, structural changes in patients with TGA were more prominent in core regions (ie, showing the highest number of altered connections) including: left hippocampus, left frontomarginal gyrus and sulcus, left middle anterior part of the cingulate gyrus and sulcus, left subcallosal gyrus, left planum polare of the superior temporal gyrus, left superior segment of the circular sulcus of the insula, left superior and transverse occipital sulci, left and right opercular part of the inferior frontal gyri, right inferior temporal gyrus, right anterior segment of the circular sulcus of the insula, right superior frontal sulcus, right parieto-occipital sulcus, and right pericallosal sulcus (Fig 2B). 


\begin{tabular}{|c|c|c|c|c|c|c|c|c|}
\hline \multirow[b]{2}{*}{ No. } & \multirow[b]{2}{*}{ Sex } & \multirow[b]{2}{*}{ Age } & \multirow[b]{2}{*}{ Precipitating Event } & \multicolumn{5}{|c|}{ Hippocampal Lesions on DTI Trace Images at the Acute Stage of TGA } \\
\hline & & & & Laterality & Number & Head & Location Body & Tail \\
\hline 1 & $\mathrm{~F}$ & 73 & No & 0 & 0 & 0 & 0 & 0 \\
\hline 2 & $\mathrm{~F}$ & 70 & Stressful event & Bilateral & 2 & 0 & 2 & 0 \\
\hline 3 & M & 69 & No & Bilateral & 2 & 1 & 0 & 1 \\
\hline 4 & $\mathrm{~F}$ & 76 & Stressful event & 0 & 1 & 1 & 0 & 0 \\
\hline 5 & $\mathrm{~F}$ & 56 & No & Right & 1 & 1 & 0 & 0 \\
\hline 6 & $\mathrm{~F}$ & 61 & Stressful event & Bilateral & 4 & 1 & 0 & 2 \\
\hline 7 & $\mathrm{~F}$ & 65 & Physical activity & 0 & 0 & 0 & 0 & 0 \\
\hline 8 & $\mathrm{~F}$ & 62 & No & 0 & 0 & 0 & 0 & 0 \\
\hline 9 & $\mathrm{~F}$ & 67 & Stressful event & 0 & 0 & 0 & 0 & 0 \\
\hline 10 & $\mathrm{~F}$ & 80 & Stressful event & Right & 1 & 1 & 0 & 0 \\
\hline 11 & $\mathrm{~F}$ & 62 & Stressful event & 0 & 0 & 0 & 0 & 0 \\
\hline 12 & $M$ & 57 & Physical activity & Bilateral & 3 & 0 & 2 & 1 \\
\hline 13 & $\mathrm{~F}$ & 40 & Physical activity & 0 & 0 & 0 & 0 & 0 \\
\hline 14 & $M$ & 69 & No & Bilateral & 2 & 0 & 2 & 0 \\
\hline 15 & $\mathrm{~F}$ & 70 & Stressful event & Bilateral & 3 & 2 & 0 & 1 \\
\hline 16 & M & 43 & Physical activity & Bilateral & 2 & 2 & 0 & 0 \\
\hline 17 & $\mathrm{~F}$ & 78 & Physical activity & Left & 1 & 0 & 0 & 1 \\
\hline 18 & $\mathrm{~F}$ & 63 & No & Bilateral & 2 & 0 & 0 & 2 \\
\hline
\end{tabular}
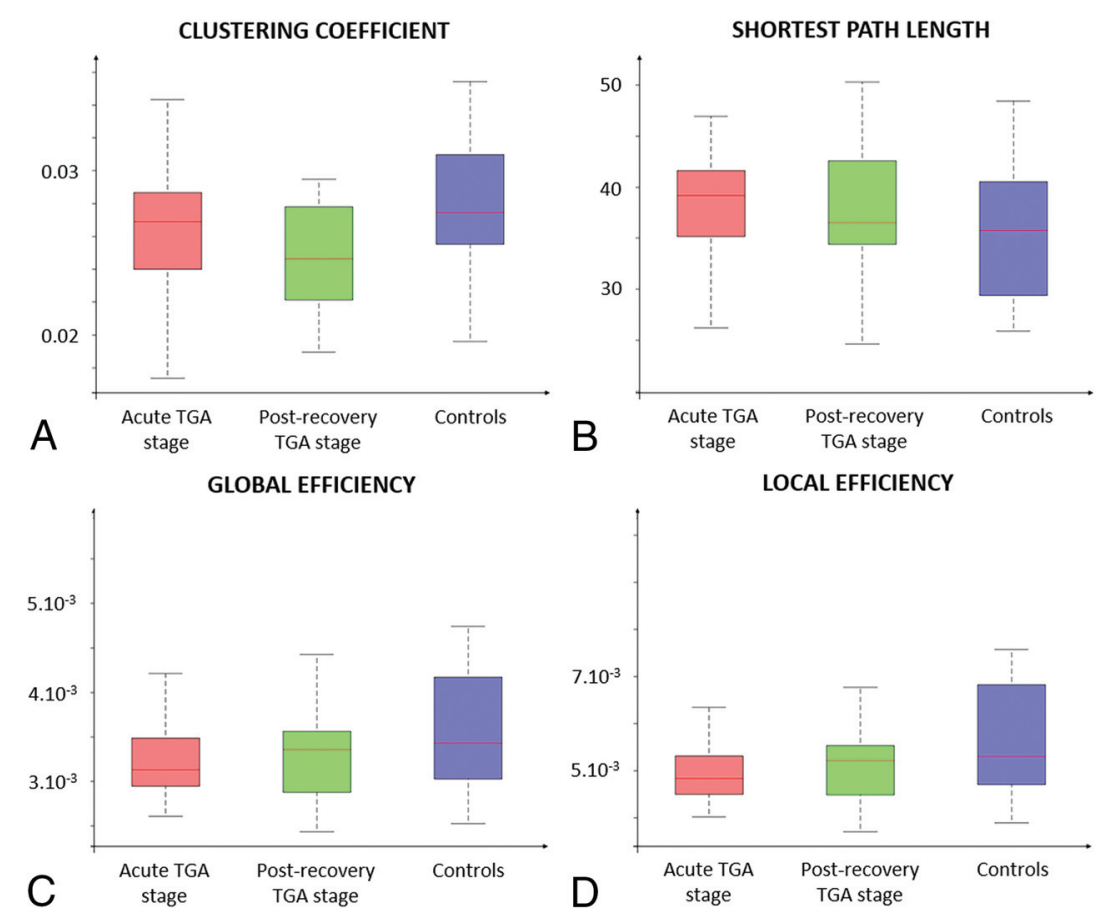

FIG 1. Global properties of the structural connectome based on normalized values of number of fibers comparing patients with TGA at acute and post-recovery stages with healthy controls. The normalization was done by dividing the number of fibers by the sum of ROI surface or volume. Clustering coefficient, shortest path length, global efficiency and local efficiency metrics were compared between groups. For each TGA stage, the global network metrics did not significantly differ between patients with TGA and controls.

NBS Comparing Patients with TGA at Acute and Delayed Stages. No statistical difference in structural connectivity was observed between acute and delayed stages in patients with TGA.

\section{Cortical Thickness and Subcortical Volumetry}

In comparison with controls, lower cortical thickness was observed in patients with TGA at the acute stage in the following cortical regions: left and right frontomarginal gyrus and sulcus, left orbital gyri, left transverse frontopolar gyri and sulci, left middle-anterior part of the cingulate gyrus and sulcus, left lateral orbital sulcus, left posterior-ventral cingulate gyrus, left subcallosal gyrus, left lateral occipito-temporal gyrus, right long insular gyrus and central sulcus of the insula, right triangular part of the inferior frontal gyrus, and right opercular part of the inferior frontal gyrus (Online Figs 1 and 2). No betweengroup difference was observed in subcortical regions.

\section{Longitudinal Follow-Up at the Delayed Post-recovery TGA Stage} All the structural abnormalities observed in patients with TGA (ie, decreased structural connectivity and decreased cortical thickness and subcortical volumetry) remained completely stable at the delayed stage, in each patient with TGA.

\section{DISCUSSION}

This is the first study evaluating NBScorrected structural connectome and cortical thickness in TGA at both acute and postrecovery stages. We found lower structural connectivity and cortical thickness in some core regions of the limbic network in patients with TGA. This preliminary study highlights the potential value of advanced MR imaging to improve our understanding of TGA. Our findings and those of the other available studies may be of use to determine the most relevant neuropsychological tests in patients with TGA, potentially reducing the time and cost of future research protocols. 


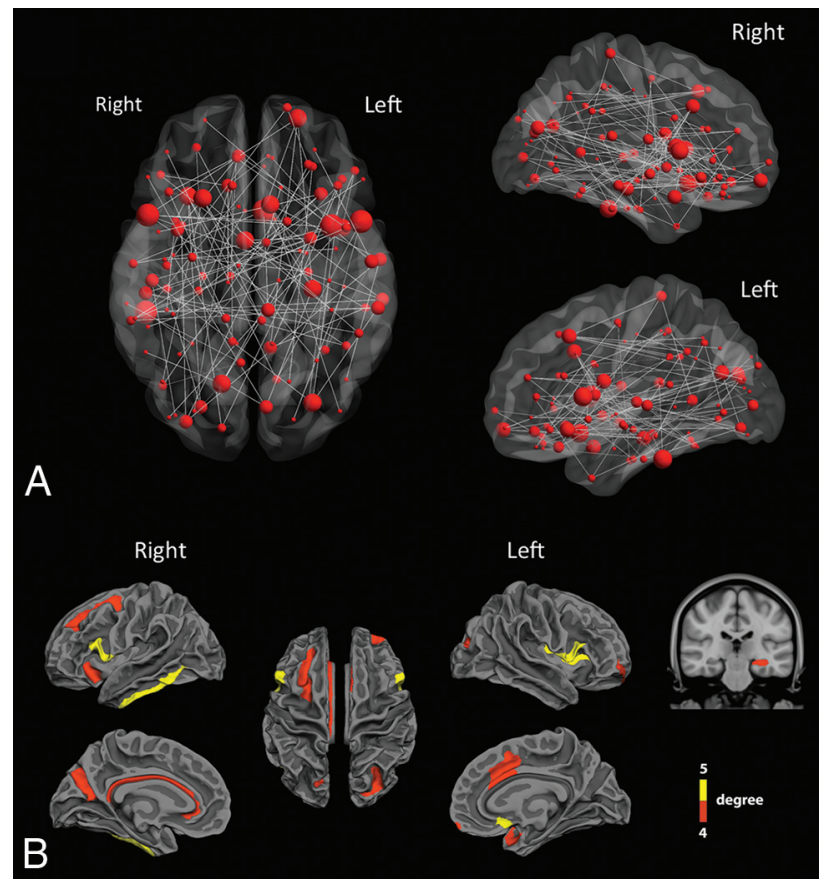

FIG 2. Altered structural connections in patients with TGA. A, Structural connectivity differences between controls and patients with TGA at acute phase. The gray lines mean significant decrease of connectivity in patients compared with controls. The red dots represent the location of ROls and their size is proportional to the number of significant connections. A network of 113 ROls and 114 connections had signifcantly decreased structural connectivity in patients with TGA $(P<.05$ NBS corrected, $t$-values ranging from 3.03-8.73). B, In this widespread network, the cortical and subcortical regions (nodes) with a number of significantly altered connections between patients and controls greater than 4 were mainly located in inferior and mesial temporal, cingulate, and frontal cortices.

Of note the topologic organization of the structural connectome was not altered in patients with TGA. Rather than a global disorganization, our study suggests altered connectivity of a specific network in patients with TGA. Such findings are in agreement with neuropsychological studies demonstrating a selective disorder of episodic memory in TGA without impairment of other components of memory. ${ }^{18,19}$ Indeed, as suggested by previous SPECT, PET, tract-based spatial statistics, and fMRI studies, ${ }^{10,12-14,20-22}$ the present study leads to the concept of TGA as a network disease.

We found structural alterations in several brain regions that are all known to be involved in different stages or components of memory processing, including: hippocampus, temporal lobe, orbitofrontal cortex, and parieto-occipital sulcus or cingulum. Indeed, most of these regions are directly involved in memory processing, in strategic or executive-based processing of mnemonic information meant to ensure its successful encoding or retrieval. The orbitofrontal and dorsal anterior cingulate cortices are involved in value-based modulations and temporal contextprocessing during memory (the ability to replace events or their aspects in the right time). ${ }^{23,24}$ The frontomarginal and dorsal anterior cingulate regions are involved in prospective memory and future-thinking, ${ }^{25,26}$ as well as in inhibitory control during encoding and retrieval. The ventral lateral frontal regions tap into incidental and intentional memory encoding. ${ }^{27}$ The retrosplenial cortex is also assumed to perform the translation between egocentric and allocentric representations formed into the hippocampus, mostly during navigation. ${ }^{28}$

Interestingly, some brain regions with decreased connectivity are also involved in meta-cognitive processing. The dorsal anterior cingulate regions, together with the subcallosal cortex and the insula are all involved in mnemonic monitoring processes such as self-assessment of encoding or retrieval and confidence in self-performance. ${ }^{29,30}$ This network appears critical to ensure successful memory given its connections to frontal and medial temporal regions, parahippocampal cingulate bundle and fornix. ${ }^{31}$ Overall, this network allows for strong interactions between memory processing and meta-cognitive processes so that associative regions could interact with interoceptive cortex and salience regions to promote accurate judgment of self-memory performance $^{32}$ as well as self-projection in different spatial and temporal dimensions. $^{26}$

To compare the cortical thickness between patients and controls we performed an ROI analysis, which is potentially more sensitive than the voxel-based approach previously used in patients with TGA. $^{8}$ Decreased cortical thickness involved the orbitofrontal, cingulate, and inferior temporal cortices of patients with TGA. Interestingly, several regions of the limbic network showed alterations of both structural connectivity and cortical thickness (left subcallosal gyrus, left lateral orbital sulcus, left middle anterior cingulate cortex, and right opercular inferior frontal gyrus), further strengthening our results.

Our study has some limitations. There was no patient scanned during the hyperacute phase. Because TGA is both rare and short-lived, neuroimaging studies performed during an attack of amnesia are also rare. In this pilot study, the reference standard for the diagnosis of TGA was based on the Hodges and Warlow criteria that are widely used in the clinical practice $^{1}$ and completely recovered after the event. Further studies are required to correlate the structural abnormalities with neuropsychological assessments of cognitive and emotional functions.

\section{CONCLUSIONS}

Compared with controls, both structural connectivity and cortical thickness were significantly decreased in the limbic network in patients with TGA. This preliminary study highlights the potential value of advanced MR imaging to improve our understanding of TGA and may be of use to plan further prospective studies.

\section{REFERENCES}

1. Hodges JR, Warlow CP. Syndromes of transient amnesia: towards a classification. A study of 153 cases. J Neurol Neurosurg Psychiatry 1990;53:834-43 CrossRef

2. Jager T, Bazner $\mathrm{H}$, Kliegel $\mathrm{M}$, et al. The transience and nature of cognitive impairments in transient global amnesia: a meta-analysis. J Clin Exp Neuropsychol 2008;31:8-19 CrossRef

3. Bartsch T, Alfke K, Deuschl G, et al. Evolution of hippocampal CA1 diffusion lesions in transient global amnesia. Ann Neurol 2007; 62:475-80 CrossRef 
4. Weon YC, Kim JH, Lee JS, et al. Optimal diffusion-weighted imaging protocol for lesion detection in transient global amnesia. AJNR Am J Neuroradiol 2008;29:1324-28 CrossRef

5. Sartoretti T, Sartoretti E, Binkert C, et al. Diffusion-weighted zonal oblique multislice-epi enhances the detection of small lesions with diffusion restriction in the brain stem and hippocampus: a clinical report of selected cases. AJNR Am J Neuroradiol 2018; 39:1255-59 CrossRef

6. Nakada T, Kwee IL, Fujii Y, et al. High-field, T2 reversed MRI of the hippocampus in transient global amnesia. Neurology 2005;64: 1170-74 CrossRef

7. Park KM, Han YH, Kim TH, et al. Pre-existing structural abnormalities of the limbic system in transient global amnesia. J Clin Neurosci 2015;22:843-47 CrossRef

8. Kim HC, Lee BI, Kim SE, et al. Cortical morphology in patients with transient global amnesia. Brain Behav 2017;7:e00872 CrossRef

9. Baron JC, Petit-Taboue MC, Le Doze F, et al. Right frontal cortex hypometabolism in transient global amnesia. A PET study. Brain 1994;117:545-52 CrossRef

10. Eustache F, Desgranges B, Petit-Taboue MC, et al. Transient global amnesia: implicit/explicit memory dissociation and PET assessment of brain perfusion and oxygen metabolism in the acute stage. J Neurol Neurosurg Psychiatry 1997;63:357-67 CrossRef

11. Guillery B, Desgranges B, de la Sayette V, et al. Transient global amnesia: concomitant episodic memory and positron emission tomography assessment in two additional patients. Neuroscience Letters 2002;325:62-66 CrossRef

12. Jang JW, Park YH, Park SY, et al. Longitudinal cerebral perfusion change in transient global amnesia related to left posterior medial network disruption. PloS One 2015;10:e0145658 CrossRef

13. Peer M, Nitzan M, Goldberg I, et al. Reversible functional connectivity disturbances during transient global amnesia. Ann Neurol 2014;75:634-43 CrossRef

14. Zidda F, Griebe M, Ebert A, et al. Resting-state connectivity alterations during transient global amnesia. NeuroImage Clin 2019;23: 101869 CrossRef

15. Rubinov M, Sporns O. Complex network measures of brain connectivity: uses and interpretations. NeuroImage 2010;52:1059-69 CrossRef

16. Achard S, Salvador R, Whitcher B, et al. A resilient, low-frequency, small-world human brain functional network with highly connected association cortical hubs. J Neurosci 2006;26:63-72 CrossRef

17. Zalesky A, Fornito A, Bullmore ET. Network-based statistic: identifying differences in brain networks. NeuroImage 2010;53:1197-207 CrossRef
18. Bartsch T, Deuschl G. Transient global amnesia: functional anatomy and clinical implications. Lancet Neurol 2010;9:205-14 CrossRef

19. Guillery-Girard B, Quinette P, Desgranges B, et al. Long-term memory following transient global amnesia: an investigation of episodic and semantic memory. Acta Neurol Scand 2006;114:32933 CrossRef

20. LaBar KS, Gitelman DR, Parrish TB, et al. Functional changes in temporal lobe activity during transient global amnesia. Neurology 2002;58:638-41 CrossRef

21. Chung YA, Jeong J, Yang DW, et al. A Tc-99m SPECT study of regional cerebral blood flow in patients with transient global amnesia. NeuroImage 2009;47:50-55 CrossRef

22. Park KM, Lee BI, Kim SE. Is transient global amnesia a network disease? Eur Neurol 2018;80:345-54 CrossRef

23. Frank LE, Preston AR, Zeithamova D. Functional connectivity between memory and reward centers across task and rest track memory sensitivity to reward. Cogn, Affect Behav Neurosci 2019; 19:503-22 CrossRef

24. Duarte A, Henson RN, Knight RT, et al. Orbito-frontal cortex is necessary for temporal context memory. J Cogn Neurosci 2010;22: 1819-31 CrossRef

25. Volle E, Gonen-Yaacovi G, Costello Ade L, et al. The role of rostral prefrontal cortex in prospective memory: a voxel-based lesion study. Neuropsychologia 2011;49:2185-98 CrossRef

26. Burgess PW, Gonen-Yaacovi G, Volle E. Functional neuroimaging studies of prospective memory: what have we learnt so far? Neuropsychologia 2011;49:2246-57 CrossRef

27. Fletcher PC, Henson RN. Frontal lobes and human memory: insights from functional neuroimaging. Brain 2001;124:849-81 CrossRef

28. Lambrey S, Doeller C, Berthoz A, et al. Imagining being somewhere else: neural basis of changing perspective in space. Cereb Cortex 2012;22:166-74 CrossRef

29. Hebscher M, Barkan-Abramski M, Goldsmith M, et al. Memory, decision-making, and the ventromedial prefrontal cortex (vmPFC): the roles of subcallosal and posterior orbitofrontal cortices in monitoring and control processes. Cereb Cortex 2016;26:4590-601 CrossRef

30. Vaccaro AG, Fleming SM. Thinking about thinking: a coordinatebased meta-analysis of neuroimaging studies of metacognitive judgements. Brain Neurosci Adv 2018;2:239821281881059 CrossRef

31. Bubb EJ, Kinnavane L, Aggleton JP. Hippocampal - diencephalic cingulate networks for memory and emotion: An anatomical guide. Brain Neurosci Adv 2017;1:239821281772344 CrossRef

32. Fleming SM, Dolan RJ. The neural basis of metacognitive ability. Phil Trans R Soc B 2012;367:1338-49 CrossRef 